\title{
IN AND OUT OF SYNC: RITMOS DE TRADUÇÃO EM PERFORMANCE E DANÇA
}

\author{
Gabriele Brandstetter* \\ Freie Universität Berlin
}

\author{
Tradução de: \\ Mariana Cristine Hilgert** \\ Universidade Federal de Santa Catarina
}

Evelyn Schuler Zea***

Universidade Federal de Santa Catarina

\begin{abstract}
* Gabriele Brandstetter é professora titular do Departamento de Teatro e Dança da Freie Universität Berlin - Universidade Livre de Berlim. Formada em filologia e em estudos literários, ela é conhecida e reconhecida internacionalmente como pioneira em estudos de dança e na transformação deste campo de estudos em uma disciplina acadêmica. Nesta área, publicou, ReMembering the Body (2000), Tanz als Anthropologie (2007), Dance (and) Theory (2013), Poetics of dance (2015), entre outros. Desde 2008, é co-diretora do Centro Internacional de Pesquisa "Interweaving Performance Cultures”, em Berlim. Email: theater-tanz@fu-berlin.de

** Mariana Cristine Hilgert possui graduação em Jornalismo pela Universidade Federal de Santa Catarina (2009) e mestrado em Ciências da Tradução (Übersetzungswissenschaft) pela Ruprecht-Karls-Universität Heidelberg, Alemanha, tendo sido bolsista da Konrad Adenauer Stiftung (KAS) de 2010 a 2013. Atualmente, é doutoranda no Programa de Pós-Graduação em Estudos da Tradução (PGET) da Universidade Federal de Santa Catarina e bolsista Capes. E-mail: marianahilgert@gmail.com

${ }^{* * *}$ Evelyn Schuler Zea tem graduação em Ciências Humanas (Etnologia, Filosofia, Teoria Literária) pela Universidade de Basel (1997), mestrado em Etnologia pela Universidade de Basel (1999) e doutorado em Antropologia Social pela Universidade de Bern (2006), reconhecido pela Universidade de São Paulo (2010). É professora no Departamento de Antropologia da Universidade Federal de Santa Catarina e docente no Programa de Pós-Graduação em Antropologia Social (PPGAS-UFSC) e no Programa de Pós-Graduação em Estudos da Tradução (PGET-UFSC). Florianópolis, Santa Catarina, Brasil. E-mail: evelynsz@gmail.com
\end{abstract}


Resumo: Este artigo focaliza questões relativas a sincronização de movimentos; coreografia e performance, bem como a técnicas corporais que abordam temáticas de integração e perturbação (de-sincronização, "Out of Sync"). Partindo de questões políticas e sociais da atualidade, levantadas pela transposição de fronteiras e por movimentos migratórios, o presente artigo relaciona o tema das "sincronizações" a reflexões sobre tradução e entrelaçamentos de culturas de movimento "a serem traduzidos". Situações de dentro e fora de sincronia, "In and Out of Sync", bem como dinâmicas e ritmos de movimento, expostos ao final como um "rite de passage" entre vida e morte, são apresentados a partir de exemplos de diferentes performances de dança, nas quais formas divergentes de conhecimento corporal se sincronizam com técnicas de movimento. À luz da instalação "Orfeu" (2015), de Susanne Kennedy, coloca-se em discussão a relevância da zona liminar do "entre", "In between" e da intrusão (com base na teoria de Jean Luc Nancy sobre o intruso, "L'Intrus") no que diz respeito à des-sincronização.

Palavras-chave: Des-sincronizações de movimentos. Transposição de fronteiras. Tradução. Migração. Detour/rodeio. Entrelaçamento de culturas de movimento na dança. A figura do intruso („L'Intrus“).

\title{
IN AND OUT OF SYNC RHYTHMS OF TRANSLATION IN PERFORMANCE AND DANCE
}

\begin{abstract}
This article focuses on questions of synchronization of movements; choreographies and performances as well as body techniques concerned with integration and disturbance issues (de-synchronization, "Out of Sync"). Starting from the current political and social questions raised by the crossing of borders and migration, the article relates the subject of "synchronization" to some considerations about translation and the interweaving of cultures of movement "to be translated". Situations of "In and Out of Sync" - and the dynamics and rhythm of movement, finally presented as "rite de passage" between life and death - are analyzed upon examples of different dance performances, where diverging forms of body knowledge and techniques of movement are synchronized. The relevance of the time zone of "In between" and the intrusion ("L'Intrus" upon Jean Luc Nancy's theory) with regard to des/synchronization is put up for discussion in the light of Susanne Kennedy's Installation "Orfeo" (2015). Keywords: De/synchronization of movements. Bordercrossing translation. Migration. Detour. Interweaving movement cultures in dance. The figure of the intruder ("L'Intrus").
\end{abstract}


Permitam-me começar por um assunto que ganhou força nas últimas semanas: a questão das "fronteiras". A palavra "tradução" quer dizer "levar para o outro lado", "cruzar fronteiras".
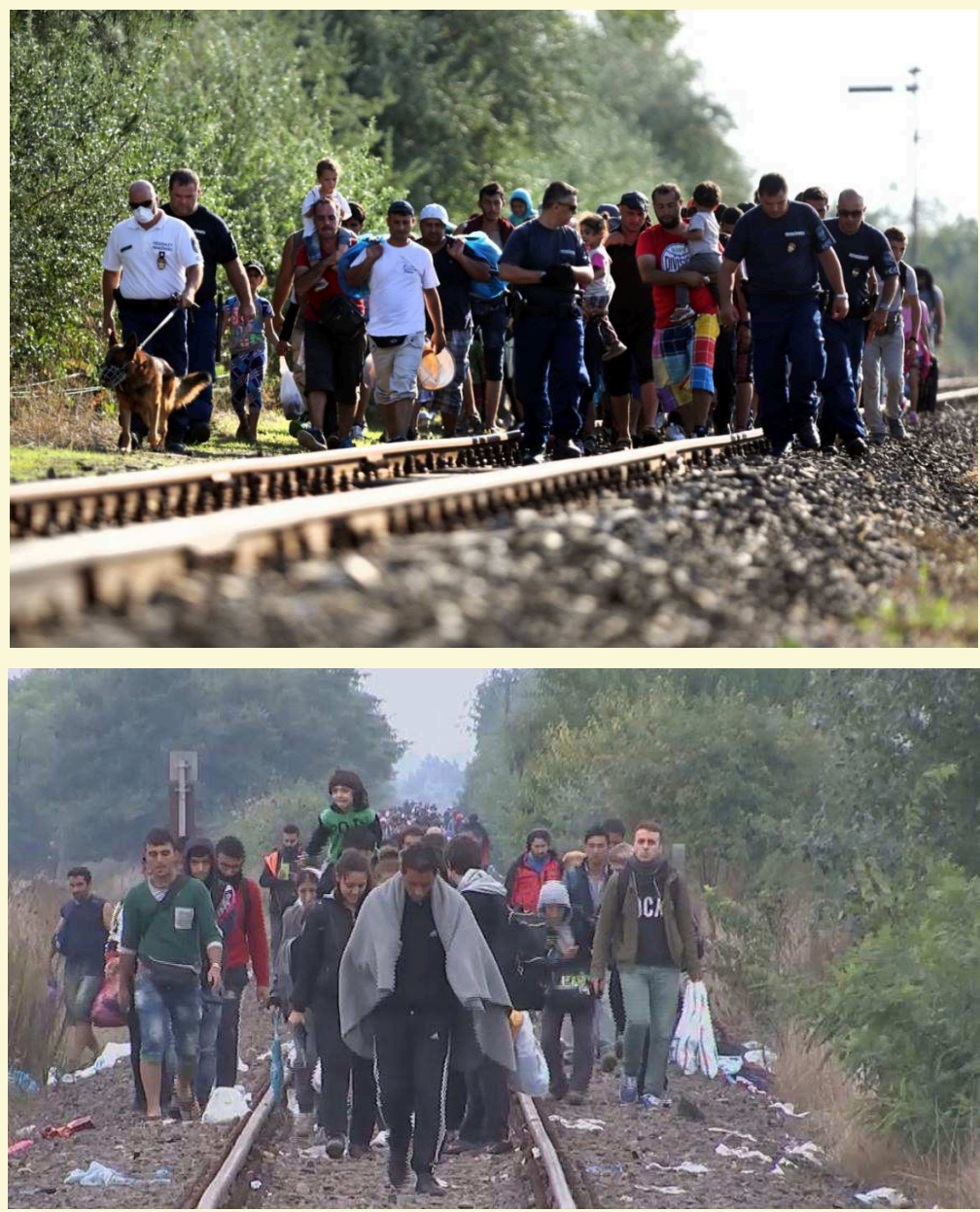

Fig. 1a \& 1b: Refugiados numa estrada de ferro na Hungria. ${ }^{\circledR}$ imago/ZUMA Press 
O que acontece quando fronteiras são abertas, inundadas - ou (novamente) fechadas e bloqueadas? Tome-se o caso da Alemanha - e da Europa, no geral -, no Outono de 2015. Milhares de pessoas, desalojadas por guerras, conflitos civis e um caos generalizado, cruzam as fronteiras - segundo estimativas, os números chegaram a um milhão apenas neste ano. O grande fluxo de pessoas e o intenso movimento migratório questionam as fronteiras não apenas enquanto demarcações territoriais (aqui acaba a Áustria, aqui começa a Alemanha), mas também enquanto demarcações e limites sociais, espaciais e corporais. Gestos de boas vindas se contrapõem a atos violentos de resistência. A capacidade dos países em prover, de última hora, alimentação, abrigo e "integração", chegou ao seu limite - é o que comentaristas políticos não cansam de nos contar.

Será que a vida cotidiana está fora do ritmo? Fora de sincronia (out of sync)? A opinião pública se encontra diante do desafio de absorver esse intenso fluxo migratório. Há uma necessidade de sincronização em níveis diversos - micro-sincronizações, temporal, física e interpessoal.

No ar, paira um sentimento de grande incerteza, que assume, para muitas pessoas, uma dimensão existencial, mais imaginária do que "real". No entanto, é justamente aí onde as fronteiras se alteram. Isto, por sua vez, levanta grandes questionamentos para a política e para as ciências em diversas áreas. Helga Nowotny, em seu novo livro The cunning of uncertainty, aborda uma questão chave dos estudos sociais de teorias da ciência atuais, relativa à busca por segurança e aos recursos produtivos da "incerteza". ${ }^{1}$

As questões que trago a seguir não se dirigem, contudo, em primeiro plano, a esferas sociais da sincronização de movimentos, mas à dança, que pode ser considerada um meio destacado, no qual processos de sincronização e dessincronização dentro de trajetórias temporais e espaciais do movimento são pesquisados e tematizados em coreografias.

Inicialmente, gostaria de apresentar uma pequena cena que evoca este "out of sync", este estar "fora de sincronia", antes de me referir a questões teóricas mais gerais sobre entrelaçamentos e rit- 
mos relativos à sincronização de movimentos. A cena em questão é do romance de Theodor Fontane Antes da tempestade (Vor dem Sturm $)^{2}$. Na estória, lembranças da guerra napoleônica na Rússia entram em fricção com o colapso psicológico do protagonista - estão, portanto, "out of sync", fora de sincronia.

Confuso e desamparado, Lewin, o protagonista do romance, busca um caminho para sair de Berlim. Ao chegar num vilarejo, se depara com a hospedaria "Zur Neuen Welt" (Novo Mundo). Ele ouve a música, vê os jovens casais dançando no salão e, do lado de fora, observa os movimentos. "Ele acompanhava o ritmo com o pé direito e se regozijava dançando. 'Quem não gostaria de estar ali!'” pensa Lewin. No entanto, sua inquietude interior o leva a seguir adiante. Ao fundo, ele ainda escuta a música escocesa dançante, enquanto retoma a marcha do seu passo. Mas ele não consegue se sincronizar, pois o compasso dançante da música invade o compasso rígido de sua marcha. Ele está fora de sincronia, "out of sync". "Lewin, enquanto prossegue, tenta retomar o ritmo de sua andada. Funciona por um tempo, mas o ritmo da dança é mais forte. Saindo, volta e meia, do ritmo forçado de sua marcha, ele caminhava ao longo da estrada de choupos, numa alternância curiosa de dança e marcha." 3

Seus passos escapam do compasso regular. O movimento do salto da dança escocesa, ("Écossaise") interrompe o compasso de sua marcha. E este movimento dessincronizado o leva àquela fronteira, àquela barreira, onde ele rememora a guerra e a derrota do grande exército de Napoleão. Mas também as imagens deste "terrível encontro" sucumbem diante daquela música. Os ritmos ligeiros e dançantes da festa do vilarejo sobrepõem-se ao compasso da marcha da tropa e à coreografia da (já perdida) guerra.

Como podemos entender rotas e encontros em termos de dessincronização, levantados atualmente por processos migratórios no mundo todo? Como podemos descrever as transformações dos espaços, dos coletivos e das relações sociais enquanto coreo-grafia? Através de uma leitura crítica do conceito de lines (linhas), de Tim Ingold, Evelyn Schuler propõe substituir e repensar a ideia da linha 
(reta), - a qual permanece sempre estática, gerando exclusão pelo conceito de rodeio (Umweg), cunhado por Hans Blumenberg ${ }^{4}$.

\section{Deslocando, viajando e migrando}

Estabelecer uma relação entre os atos de dançar, viajar e migrar implica em olhar para formas de movimentos corporais em diferentes níveis e perguntar, assim, por que e como os movimentos influenciam, iniciam, previnem ou transformam a dinâmica de outros movimentos. É um campo complexo. Viagem e migração são empreendidas por razões, motivações e durações distintas. Enquanto o turismo e as viagens "recreacionais" aumentaram enormemente nas últimas décadas, tornando-se um dos maiores fatores de impacto econômico (e impacto enormemente ecológico) no mundo, formas de migração involuntária, deslocamentos forçados e o cruzar de fronteiras também acontecem diariamente, motivados por crises políticas e/ou questões de desenvolvimento econômico. Trabalhadores migrantes, em suas diásporas mais ou menos temporárias, enviam dinheiro às suas famílias em larga escala, no mundo todo, todos os dias. Enquanto o movimento de viajar parece se basear num sentido de partidas e retornos, no caso de movimentos migratórios, a ideia do "retorno" costuma ficar em aberto. Disto resultam avaliações opostas acerca destes movimentos globais, refletidas na opinião pública. Enquanto o ato de viajar costuma ser altamente valorizado, tematizado em conversas, ansiado e até mesmo exigido nos negócios e por empresas, a migração, por sua vez, ainda é raramente entendida em termos de diversidade e bagagem cultural, mas, antes, enquanto uma deficiência, uma força desestabilizadora e um desafio para o aparato social.

A dança e os dançarinos fazem as duas coisas: eles viajam e migram em e entre "culturas". Seus movimentos globais são formados, influenciados, possibilitados e, algumas vezes, impedidos pela 
mesma lógica/logística, por agendas políticas que se transformam, por meio de convites (institucionais), políticas de visto etc. A(s) cena(s) da dança contemporânea encontram-se profundamente conectadas com estruturas econômicas que facilitam turnês e rodeios (tours e de-tours), permitindo artistas a cooperarem globalmente e transculturalmente, e dando início a reuniões e encontros. Ao mesmo tempo, estas condições encontram apoio em produções de dança contemporânea: a dança e a performance tornaram-se um local privilegiado para refletir criticamente sobre as condições que levam as pessoas a estarem "em movimento". De que maneira as contínuas transformações globais têm efeito sobre nossos corpos ao lidarmos com essas novas condições?

Corpos que viajam e corpos que migram são, com frequência, corpos em crise, colocados em situações instáveis, em infraestruturas e constelações sociais pouco familiares, usualmente controlados por autoridades enquanto se movem, regulados e limitados em seus caminhos e decisões. Corpos que viajam costumam ser, assim, corpos com um alto grau de sensibilização, que desenvolvem táticas para se adaptarem, resistirem, desapegarem e apegarem-se a hábitos, ideias e pertences pessoais. Quais são os vestígios que estes trânsitos e estas transições deixam nos corpos? Como estes vestígios re-formulam o jeito habitual desses corpos se moverem? Que tipos de histórias eles instigam? Que tipo de estruturas de poder eles revelam? De que maneira as histórias pessoais e interpessoais estão sendo formatadas por estas viagens e migrações? Como podemos encontrar e descrever linguagens (corporais) que reflitam adequadamente estas complexas relações, transformações, estas influências "subcutâneas" que os movimentos (globais) exercem sobre nosso variado vocabulário, nossas disposições, nossos gestos e nossos movimentos cotidianos?

Quais são os movimentos que emergem nas cenas coreo-políticas das culturas de dança e de performance? E como eles, por sua vez, entrelaçam-se com movimentos em ambientes urbanos e em redes midiáticas? A coreógrafa ítalo-sueca Cristina Caprioli de- 
senvolveu, em 2012, um projeto relacionado a "weaving politics" (tecendo políticas). Caprioli deu continuidade a este projeto por meio de uma "Choreo_Drift", um formato apresentado em diversas cidades da Europa, Estados Unidos e Oriente Médio durante um longo período, num singular processo de entrelaçamento de eventos de dança, performance e teoria, sobrepondo-se e interrompendo-se mutuamente - e assumindo os fios uns dos outros em lugares diferentes.

O corpo humano se constitui ele mesmo enquanto sua arena particular de movimentos e sedimentos de "entrelaçamentos". Este corpo, nele mesmo, não é apenas um. É possível questionar, a partir de Roland Barthes: "Que corpo? Temos muitos corpos: o corpo de anatomistas e fisiologistas, aquele que a ciência vê ou sobre o qual fala: este é o texto de gramáticos, críticos, comentaristas, filólogos (o fenotexto)" (1975, p.16). A isto, Barthes contrapõe o "corpo do prazer" (body of bliss) (ibid, p.16): um corpo que se dissolve, se dissipa e dissemina a si mesmo - a cena de entrelaçamentos e suas liquefações, irredutíveis à sua fisiologia. Barthes descreve uma situação que realça estes movimentos de enredamento e ressonâncias e a sobreposição de corpos (sonoros) interculturais e de mídias diversos. Sentado num bar em Tangier, Marrocos, ele vivencia a si mesmo como uma zona de trânsito, como um espaço público de múltiplos corporais: "Música, conversas, os sons de cadeiras, vidros, uma completa estereofonia, da qual um bairro em Tangiers (como descrito por Severo Sarduy) é o lugar exemplar. Aquilo também dialogou internamente comigo, e esta chamada conversa 'interior' soou como o barulho do bairro, como aquele acúmulo de vozes menores vindas de fora em minha direção: Eu mesmo era o bairro público, a feira (souk) (...)" (ibid, p.49). Será o corpo dos dançarinos, hoje em dia, tal "souk", feira? Um espaço público, onde diferentes culturas e práticas de movimento, técnicas corporais e experiências somáticas sobrepõem-se umas às outras - uma concorrência e uma não-simultaneidade polirítmica, estereofônica, multivocal e multilíngue de camadas, cujos padrões enredados continuam a gerar 
diferenças e fricções? No contexto de expectativas e experiências com as quais os dançarinos de hoje se deparam em audições, o corpo que se destaca se constitui enquanto cena de "entrelaçamentos" de várias técnicas de dança e práticas somáticas. ${ }^{5}$ Balé, dança moderna (seja a partir das técnicas de Graham, Limon ou Cunningham) e, junto a isto, técnicas de liberação, além de treino de tai chi, yoga, experiências em Body-Mind-Centering $(\mathrm{BMC})^{6}$, cursos de técnica Alexander e método Feldenkrais. Este tipo de "mix", com diferentes ênfases, costuma caracterizar o treinamento e o processo de habituação aos quais os bailarinos estão condicionados: trata-se de sincronizações do corpo a nível intra-corporal advindas de culturas de movimentos e dança muito distintas e de suas tradições, frequentemente muito antigas e padronizadas. Isto nos coloca inúmeras questões: Como as técnicas de movimento e seus exercícios de incorporação se transferem, se entrelaçam ou colidem mutuamente? Como o treino de balé e tai chi, por exemplo, se combinam ou se contradizem entre si? E de que maneira estas sincronizações micro-corporais e seus potenciais específicos tornam-se produtivos em termos de design estético e percepção?

O que enriquece as "realidades corporais" das performances de dança contemporâneas é menos a uniformização destas incorporações (embodiments) de diversas técnicas de movimento, e muito mais as suas diferenciações (individuais e locais, conforme for), com todas as suas inconsistências? Não é o aspecto reluzente das virtuosidades técnicas, mas as fraturas e as interferências daquilo que não se consegue facilmente sintetizar em um "corpo" - o cenário em si, isto é, aquilo que, performativamente, gera este “certo" corpo múltiplo enquanto local permeável, enquanto área de trânsito entre "redes" diversas e não-simultâneas, sem fixá-lo, como descreve R. Barthes. Este é o foco de pesquisas e análises em "movimentos de entrelaçamento". ${ }^{7}$

Alguns exemplos servirão para mostrar que não é possível reduzir camadas e tensões de entrelaçamentos intra-corporais a "tipos" ou "formatos" muito claros. Mais do que isso, o fato 
de estarem ambas, tanto a diferenciação quanto a des-diferenciação, se tornando relevantes em cenas individuais e trabalhos artísticos é o efeito da presença globalizada de uma cultura corporal de cursos e eventos, a partir da qual disseminam-se cursos de yoga, chi gong, flamenco, salsa, hip-hop e break-dance, além de outras técnicas e práticas de movimento pelo mundo. É um processo que tem uma história própria. Reflexões sobre "outras" práticas de corpo, e não apenas sobre a forma virtuosa e elegante, remontam à geração do Judson Dance Theater e, mais adiante, ao Modernismo na dança. Este último, apesar de se encontrar ainda sob a insígnia do Ocidente colonizador e das instâncias associadas à auto-exoticização do corpo (feminino), já se apresenta enquanto cenário de enredamentos de culturas de movimento, e seus respectivos discursos, completamente diferentes, tanto social quanto esteticamente.

Performances de dança, especialmente a partir dos anos 80 e 90, expõem-se costumeiramente, tanto em sua prática quanto em seus conceitos, como um "produto das circunstâncias", no que diz respeito às suas práticas fisicamente interdependentes e culturas de movimento diferentes.

Peça chave dos anos 90, a performance de Xavier Le Roy intitulada "Produto das circunstâncias" (1998), por exemplo, tornouse o projeto central de uma performance do "corpo contaminado" (SABISCH, 2002). O conceito de contaminação também sempre sugere "positing" (colocação/Setzung), a conotação da impureza, quando não do envenenamento; uma prática de "sincronização" enquanto prática pretendida e latente é, por outro lado, mais ampla no seu escopo, também no sentido de que os níveis de uma prática discursiva e de uma reflexão analítica estão implicados no conceito de de/sincronização. Entre os exemplos da enorme variação e divergência de fenômenos desta natureza, isto é, que envolvem um enredamento incorporado (embodied) e intra-corporal de diferentes práticas e tradições de corpo (salientadas aqui pela multiplicidade da cena performática), estão: as peças de dança do bailarino Koffi Kôkô, que, em seu trabalho, entrelaça as origens das práticas xa- 
mânicas corporais do Benin (África), seu país de origem, com as experiências de sua prática em dança moderna em Paris, ambas presentes nos padrões de movimentação e nos temas de suas peças; ${ }^{8}$

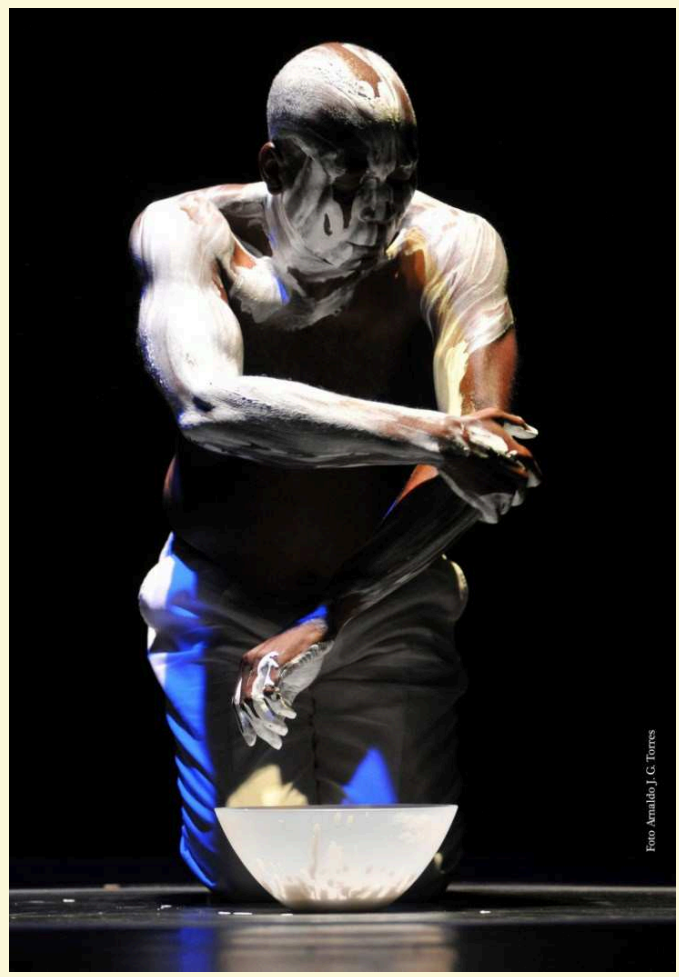

Fig. 2 : Koffi Kôkô, La Beauté du Diable, Torinodanza $2013{ }^{\circledR}$ Arnaldo-J.G. Torres

o trabalho de Pitchet Klunchun, um dançarino da Tailândia, treinado em dança Khon clássica, que, junto com o francês Jérome Bel (Pitchet Klunchun and myself),criou um duo no formato de uma performance-diálogo (lecture performance); e que, na peça "I am a Demon" (Eu sou um demônio) (2007), associa, por fim, a tradição de educação em dança Khon com sua adaptação do conceito (ocidental) de performance autobiográfica, enquanto, ao mesmo tem- 
po, subverte e transforma sua jogada com o "Eu", já presente no título. De que maneira estas performances ocupam uma zona cinzenta de transferências de práticas somáticas e daquilo que é transmitido apenas num formato autorizado, protegido enquanto uma “marca", como é o caso das práticas de Klein Technique ${ }^{\mathrm{TM} 9}$ ou dos workshops do sistema "Gaga" ${ }^{10}$ desenvolvido por Ohad Naharin?

Quais são as novas fronteiras, quais são as inclusões e as exclusões que, aqui, se tornam efetivas? É necessário mencionar, neste contexto, que tais transferências e sobreposições de diferentes práticas culturais de corpo também dizem respeito a "camadas" de inúmeros "hábitos" históricos e conceitos de movimento. Por meio delas, o corpo do dançarino se torna o local de um palimpsesto corporal - é o caso, por exemplo, de Kazuo Ohno, dançarino japonês e pioneiro do Butoh, ao combinar, deslegitimar e reformular por completo tradições ocidentais e orientais em suas performances.

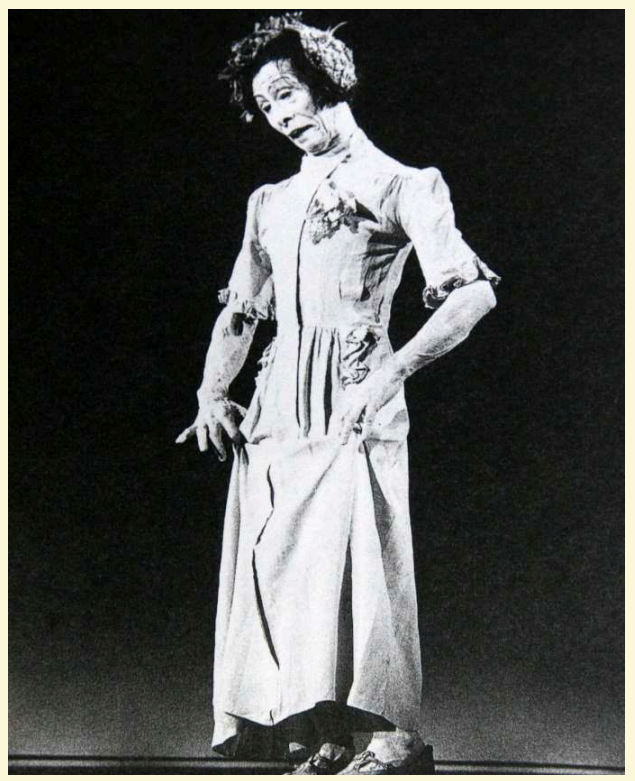

Fig. 3: Kazuo Ohno, Admiring La Argentina, 1977, http://theredlist.com 
Em Admiring la Argentina, ele incorpora, aos 78 anos, o estilo de dança da estilizada "dança espanhola", executada pela célebre bailarina da década de 20 "La Argentina" durante sua turnê internacional, incluindo também o Japão. Ao mesmo tempo, Ohno mescla este hábito do "Flamenco" com modos de fluxo de movimento vindos da dança expressionista alemã, introduzidos aos japoneses por bailarinos como M. Ito e Ogai Mori, que estudaram e se apresentaram na Europa. Enquanto professor de educação física numa escola japonesa, Ohno aprendeu, além disso, uma prática física apropriada, e em seu encontro com Tatsumi Hijikata, tomou conhecimento da abordagem radical de rebelião corporal deste último, a qual se dá por meio do "Ankoku Butoh". Ohno transformou este conceito de expressividade através de uma abordagem idiossincrática do Butoh, por meio de delays e transparência lírica - uma dança de transformações que se dá por entrelaçamentos de diferentes culturas de movimento e conceitos de expressividade. Também a transposição de barreiras exercendo descriminação entre sexo, diferenças etárias do corpo e entre animado e inanimado se tornaram uma característica estrutural de seus métodos de incorporação. Kazuo Ohno é um exemplo do fato de que formas artísticas de dessincronização e resincronização intra e intercorporais de culturas de movimento e técnicas corporais, longe de conduzirem à homogeneização, estão aptas a contribuir para uma diferenciação altamente individualizada, quando não para articulações e expressões singulares da diferença. ${ }^{11}$

Hoje em dia, estes desenvolvimentos também levam a maiores desdobramentos em questões relacionadas a racismo, identidades nômades, souvenirs de viagem e deficiências ${ }^{12}$, perpassando, ao mesmo tempo, o terreno da diferença sexual a partir de transformações corporais. Quais são, assim, as mudanças (alterações, transformações) que acontecem quando um dançarino (mulher ou homem) passa por um processo de transgenização? Como isto afeta a qualidade de seus movimentos? Estas questões serão analisadas como uma forma de "etnografia queer" incorporada (embodied "queer etnography”) por An/(J)na Kaler, em uma série planejada 
de performances intituladas O.T. (as letras iniciais das palavras alemãs para "sem título").

\section{Fronteiras, áreas fronteiriças, passagens}

Uma visita a áreas fronteiriças, a zonas limítrofes entre vida e morte: é isso o que oferece a instalação-performance Orfeu, baseada na composição de Claudio Monteverdi. Trata-se de um rito de passagem (rite de passage), no qual o observador percorre oito salas, ligadas ente si por minúsculas zonas de espera - uma trans -lation (um traduz-ir, um ir através); um ritual que, dentro de um período fixo de tempo (10 minutos em cada sala, os quais se estendem continuamente), se encaminha a uma experiência de atemporalidade e, mesmo, de eternidade, no além.

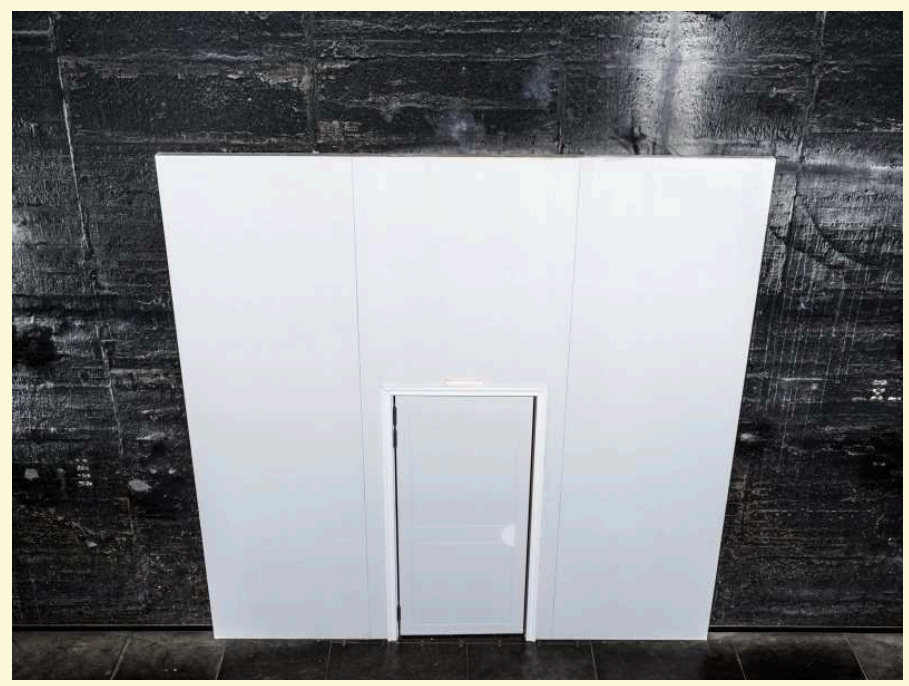

Fig.4: Orfeu, por Claudio Monteverdi. "Um exercício de morrer", dirigido por Susanne Kennedy, Bianca van der Schoot, Suzan Boogaerdt, Conjunto de solistas Kaleidoskop, ${ }^{\odot} \mathrm{JU} /$ Ruhrtriennale (Julian Röder) 
"Um exercício de morrer" - é assim que a diretora, Susanne Kennedy, denomina a instalação. ${ }^{13}$ Orfeu em busca de Eurídice somos nós, visitantes deste percurso. Deslocamo-nos em um limbo, tal qual Orfeu - num caminho de um mundo que se encontra "para além", e neste entre-reino, emerge Eurídice. Fragmentos da música de Monteverdi conectam, entre si, as etapas do percurso - e de seu rodeio. A performer de Eurídice se desloca no espaço como se controlada à distância; ela tem uma forma mascarada, semelhante a um zumbi, e se encontra presa na terra de ninguém, entre a vida e a morte, dado que Orfeu não pode libertá-la. Repetições no design do espaço, o silêncio e os fragmentos sonoros que vêm de longe, os clichês femininos das figuras de Eurídice, cujas máscaras e perucas idênticas, cuja conduta impassível e indiferente, seja ao sentar-se, ao levantar-se, ao caminhar, lhes conferem um aspecto "sintético" - tudo isto torna evidente para o espectador que a identidade está desvanecendo, "fading away".

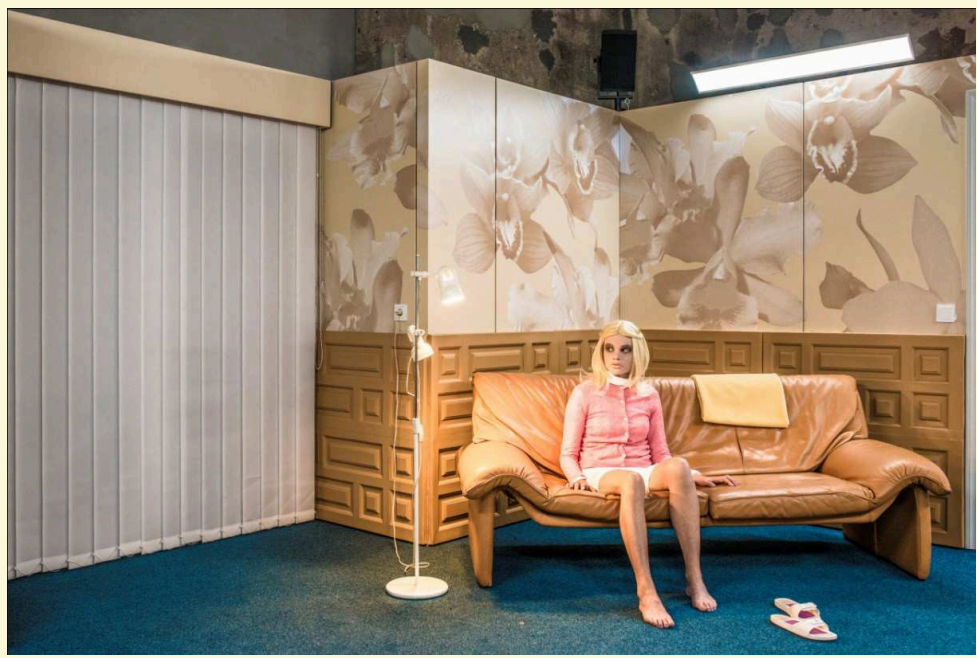

Fig.5: Orfeu, por Claudio Monteverdi. "Um exercício de morrer”, dirigido por Susanne Kennedy, Bianca van der Schoot, Suzan Boogaerdt, Conjunto de solistas Kaleidoskop, ${ }^{\odot} \mathrm{JU} /$ Ruhrtriennale (Julian Röder) 
Aquilo que outrora fora familiar torna-se estranho. À medida que o tempo é estendido, em meio a uma situação de tédio e vazio, todos são largados à própria sorte, ficando expostos a lembranças, associações e pensamentos de fuga. Apenas os olhos da Eurídice, mascarada e multiplicada, movem-se profundos, inquietantes, estranhos, por detrás das máscaras de plástico. E alguns gestos, um jeito de respirar e mexer-se no assento, como se num transe, fornecem pistas: a vida não deve demorar a acabar. Esta situação, estas figuras - e nós mesmos - caímos fora do compasso, fora de sincronia, "out of sync".

A banalidade e, ao mesmo tempo, o hiper-realismo, na proximidade dos acontecimentos, colocam o observador em uma experiência-simultânea desta transição labiríntica temporal-espacial. "Manter silêncio durante a transição" ${ }^{14}$, nos fala o programa. Somos admitidos num espaço que em que o "desprendimento", "letting go", é tanto conteúdo quanto experiência:

- "Não se agarre a esta vida por carinho e fraqueza.

- Embora você não abra mão por fraqueza

- Você não tem força para permanecer aqui” (ver programa).

Avisos relacionados à transição, inspirados no Livro tibetano dos mortos, acompanham a travessia, até o espectador, na penúltima sala, resplandecente e iluminada, ver-se diante de Orfeu. Este canta, intensamente e muito próximo a mim, sua ária de permanência - mas apenas por um momento, que se sente muito breve. Em seguida encontramo-nos com outros no "leito da morte", sobre o qual Eurídice se encontra deitada. Enquanto isso, alguns fragmentos de lembrança tremulam pela tela. "A duração deste estado é incerta" (citação do programa).

A estrutura desta instalação é complexa, e a travessia por entre as salas de fronteira acaba por gerar um alto potencial afetivo e qualidades transformativas. As zonas de fronteira encontram-se num cenário fictício: a configuração do ambiente convida o observador a relacionar o mito de Orfeu e Eurídice a suas próprias experiências e expectativas sobre a morte (ou sobre atos de desprendimentos/despedidas). Amparado ora pelo silêncio, ora pela 
música que é composta de sons estendidos (os compositores rearranjaram a partitura de Monteverdi com a ajuda de um algoritmo, estendendo-a para a duração de 199,5 horas), o espectador adentra um estado no qual a experiência da zona limítrofe é transferida ao seu interior; seja lá como for imaginado, ele se percebe num estado de passagem antecipado entre vida e morte. E a ressonância - ou a resistência - em relação àquele período de tempo, semelhante a um laço infinito, na qual as "figuras de Eurídice", já mergulhadas num estado de morto-vivo, sentam e esperam, é acompanhada por um sentimento de transformação e incerteza. Seria esta uma insegurança produtiva? ${ }^{15}$ Trata-se de um estado de desconforto, um deslocamento das fronteiras que atravessam o corpo. As performers quase não se movem - permanecem, por exemplo, sentadas no sofá. Aí elas são, quase imperceptivelmente, atravessadas por micro movimentos no torso, diafragma, na espinha dorsal (spine), como se o tônus vital esvanecesse gradativamente, e o corpo fosse perdendo a força. Esta presença fugaz, que é, na verdade, mais perceptível enquanto ausência, afeta os sentidos do observador num nível proprioceptivo e cinestésico. As fronteiras se alteram entre o interno e o externo, entre o eu e o outro (no espaço) - entre a(pós)vida e a proximidade da morte. Uma transição!

No ensaio “O intruso" (L'Intrus) ${ }^{16}$, o filósofo Jean-Luc Nancy reflete sobre o grande tema do "intruso", do invasor, como uma experiência de invasão e intrusão da morte em sua vida, em seu corpo.

Assim, o estrangeiro múltiplo que faz intrusão na minha vida (minha pequena vida, esfalfada, por vezes escorregando num mal-estar à beira de um abandono apenas atordoado) não é outro que a morte, ou antes a vida / a morte. (2008 [2000], p.14). ${ }^{17}$

Nancy escreve em virtude de um transplante de coração ao qual havia se submetido recentemente. É uma questão de vida e de morte, de receber o "intruso" em seu corpo, da impossibilidade de 
diferenciar o "próprio" do "estranho" - o transplante, a imunidade e reação de defesa do corpo (ibid, p.18). Nancy discute o tema do "intruso" no nível do seu próprio corpo, com suas fronteiras e seus limites que se transformam constantemente. Este é um tema que desafia permanentemente nossa vida social e corporal. Quem é um "intruso", quando, em qual território, em qual tempo-espaço? E como estas experiências - de cair fora do compasso, fora de sincronia, "out of sync", e de re-sincronização dos movimentos coletivos - transformam nossas noções sobre identidade (corporal, social, política)?

Nancy questiona se há alguma legislação geral sobre o intruso. Em seguida, responde:

Jamais houve uma só intrusão: desde que uma é produzida, ela se multiplica, ela se identifica em suas diferenças internas renovadas. (ibid, p. 18-19).

Distúrbios no ritmo cardíaco e extra sístoles e outras dessincronizações que o corpo vivencia internamente - como um coletivo no interior - se tornam modelos de uma experiência do intruso e da estranheza no nível social. Questões atuais sobre fronteiras e sua violação, sobre o abandono de sincronizações familiares, advindas de processos migratórios, sobre movimentos de fuga em todo o globo e sobre a cultura de "receptividade" (hospitalidade) são afetados pela reflexão de Nancy sobre o "intruso": "Acolher o estrangeiro, quer dizer também sua intrusão. O mais frequentemente, não se quer admiti-lo: o motivo do intruso é ele próprio uma intrusão em nossa correção moral (é mesmo um exemplo notável do "politically correct"). Entretanto, ele é indissociável da verdade do estrangeiro. Essa correção moral supõe que se receba o estrangeiro apagando no limiar sua estrangeiridade: ela quer, pois, que não o tenha absolutamente recebido. Mas o estrangeiro insiste e faz intrusão. É isso que não é fácil de receber, nem talvez de conceber..." (ibid, p. 4). 
Daí surge um paradoxo. A intrusão do estranho e o apagamento de sua estranheza no limiar da acolhida é o desafio e a base para a transposição/tradução (trans-lation) que, claro, nunca pode "dar certo". Pois os movimentos que estas chamadas fronteiras, zonas, passagens transpõem e descrevem são movimentos que demandam sincronizações, de- e re-sincronizações. Quebras, rupturas, lacunas - e rodeios/detours (!) - são partes desses processos. Na dança e na performance, os trabalhos mais relevantes vêm sendo produzidos por aqueles interessados na pesquisa e no afinar destas dessincronizações - tanto as sincronizações de movimentos coletivos, como também em experimentos que visam a processos intra-corporais de polirritmia e de concordâncias temporais e físicas. Assim trabalha, por exemplo, Thomas Hauert, com a Companhia Zoo. Em suas peças Verosimile (2002) e em Accords (2008), ele lida com conceitos que remetem a movimentos como os de um cardume. As observações sobre sincronizações num coletivo de corpos visto enquanto cardume é transposto a formações para dança. Isto se dá por meio de práticas somáticas e reconhecimento das relações cinestésicas entre os corpos e o espaço. O fato de a coesão do grupo se moldar constantemente surge como força propulsora da coreografia. Esta não é pré-fixada, mas toma forma no momento do "mover-se" conjunto: suas formas e seus limites temporais desenvolvem-se nos processos de sincronização dos movimentos de cardume (do grupo).

O trio Kneeding ${ }^{18}$ (2010), de Jefta von Dinther, é um exemplo de como os conceitos e modulações de dessincronizações do interior do corpo são ativados e transpostos à coreografia. Na criação de sua peça, ele emprega diversas práticas somáticas, sobretudo de Body Mind Centering (BMC). Os dançarinos modelam ("amassam") os seus corpos como se fossem materiais arbitrariamente maleáveis, à medida que inserem sincronizações complexas de órgãos e fluidos. Eles adentram a imaginação e ultrapassam as fronteiras do que se pode ver e sentir ("O que você não pode tocar"), chegando, por meio deste processo de criação, às decisões acerca do desenrolar da coreografia: proximidade e distância; velocidade 
e qualidade/ tensão dos movimentos. O espectador se vê confrontado com relações de natureza multitemporal (polychrone), visual e cinestésica, diferentemente ritmadas entre os corpos, o espaço e o som. Os dois experimentos de dessincronização - tanto o externo, com os coletivos, quanto o interno, envolvendo corpos que se encontram (entering bodies) - remetem a problemas atuais de nosso mundo globalizado. "O mundo está desabando", disse, recentemente o político alemão Frank Walter Steinmeyer em um discurso. Fora de sincronia, "Out of Sync". Será que, com isso, ele se referia a investigações em dessincronizações conduzidas enquanto estudos temporais e de movimentos e processos e transmissões policrônicos?

Como, a partir disso, é possível transpor, ampliar, deslocar e reestabelecer fronteiras? Quando as zonas de conflito (sejam eles o espaço social ou do corpo) são interiorizadas, quando questões de demarcação e expulsão (do intruso) irrompem violentamente na zona de conforto do cotidiano?

Nestes processos, não é possível traçar uma linha reta (no sentido de Tim Ingold). "Out of sync" implica, sempre, em cair fora de um processo de tempo e uma maneira de fazer as coisas (aparentemente) dados. "Out of sync" comporta, no entanto, a chance de uma reorientação; de um rodeio (Evelyn Schuler com Blumenberg) rumo a novas sincronizações provisórias do "corpo" social e individual (e de suas redes).

\section{Notas}

1. Sobre o sujeito atual da "incerteza", cf. também "Unsicherheit", volume publicado por Zeitschrift für historische Anthropologie , Paragrana, org. por Christoph Wulf e Jörg Zirfas, vol. 24, issue, 2015. Sobre incerteza e perspectivas, cf. também o ensaio de Gabriele Brandstetter "Tanz mit dem Unvorhersehbaren: Prognosen über Bewegungen". In: Gabriele Brandstetter, Sibylle Peters, Kai van Eikels (Org.) Prognosen über Bewegungen, Berlin, b_books, 2009, pp. 262-269. 
2. N.T.: Todos os trechos da obra de Fontane foram traduzidos por mim.

3. O trecho segue da seguinte forma: "Ele passou pelo lugar onde, pela primeira vez, sentiu a miséria do recolhimento. Nesse período, não mais pensou no desastroso encontro e permaneceu apenas junto à música, embora tivesse cessado de ouvi-la há bastante tempo. 'Apenas dançando', ele disse, 'isso é viver. Não deixe nada lhe colocar para baixo. O melhor passou por mim.'” (tradução nossa).

4. Cf., neste contexto, as reflexões de Blumenberg sobre a questão das fronteiras, relacionada à metáfora de Wittgenstein sobre o muro que circunda o pântano, tal qual descrito em suas "Philosophische Betrachtungen" (1930). Blumenberg explica esta imagem mental em sua coleção de textos curtos "Care Crosses the River", no texto sob o título de "Pântano" (Swamp). O que é crucial para ele (e para nossas observações sobre o conceito de "fronteira"), é o fato de que fronteiras nunca são desenhadas com precisão matemática. Pelo contrário, elas são manchas, e indicam um espaço intermediário onde inclusão e exclusão não são passíveis de definição exata. "O muro não pode corresponder exatamente com a borda do pântano. Aquele se encontra no entorno desse, delimitando-o, enquanto permanece de pé, sobre uma base segura, tal qual muros devem permanecer" (p.109, tradução nossa). Isto quer dizer que delinear a inexatidão de forma exata é impossível aqui, da mesma forma como o é em qualquer outro lugar" (p.110). E zonas/fronteiras não "possuem” um ponto de passagem. Elas são um ponto de passagem (ibid); cf. Hans Blumenberg: Die Sorge geht über den Fluss, Frankfurt/M., 1998, p.109 f.

5. Para uma discussão sobre filosofia/estética e práticas somáticas cf. Richard Shusterman: Somaesthetics - A Disciplinary Proposal, in The Journal of Aesthetics and Art Criticism, Vol. 57, No. 3. (Summer, 1999), ed. by the American Society for Aesthetics, Philadelphia, pp. 299-313.

6. Cf. Bonnie Bainbridge Cohen: Sensing, Feeling and Action, 3rd ed. Toronto, Contact Editions, 2012.

7. Cf. Gabriele Brandstetter, Gerko Egert, Holger Hartung (Org.): Movements of Interweaving, London, Routledge, 2016, previsto.

8. Entrevista com Johannes Odenthal em The Third Body - das Haus der Kulturen der Welt und die Performing Arts, ed. por Johannes Odenthal e Haus der Kulturen der Welt, Berlin, Theater der Zeit, 2004, pp. 45-52. 
9. Christina Kahlert: Klein Technique ${ }^{\mathrm{TM}}$ - Re-Patterning and Selbstkompetenz. Zur Integration somatischer Praktiken in die zeitgenössische Tanz(pädagogik) ausbildung, Diss., Berlin, 2015.

10. Einav Katan: Body of Knowledge. Embodied Philosophy in Gaga, Ohad Naharin's Movement Research, London, Palgrave, 2016, previsto.

11. Sobre o entrelaçamento de tradições do Butoh traditions com técnicas de dança ocidentais cf. o ensaio de Nanako Nakajima "Two Hours and One Hundred and Three Years of Dancing : Xavier Le Roy's Product of Other Circumstances and Kazuo Ohno's Butoh", em Fjord Review, Verão de 2011, Volume 1, Número 3, pp. 14-19.

12. Cf. Gabriele Brandstetter, Ann Cooper Albright (Org.): Choreographic Practices. Special Issue: Dis/abilities: The Politics of a Prefix, intellect journals, 2015, Volume 6, Número 1.

13. Martin-Gropius-Bau, Berlin, 18.09.-04.10.2015 (UA: 20.08.2015, Essen), apenas oito pessoas de por vez são admitidas na complexa estrutura de salas do Museu.

14. Citação extraída do programa: Orfeo. Nach Claudio Monteverde - eine Sterbeübung. Produzido por Ruhrtriennale e o conjunto Kaleidoskop, com coprodução de Berliner Festspiele e Toneelgroep Oostpool, 2015.

15. Cf. acima: G. Brandstetter: „Produktive Unsicherheiten. Kontingenz und Improvisation im Tanz", in: Valeriĩ Savchuk, Christoph Wulf (Org.), Neopredelennost' kak vyzov : media, antropologiia, èstetika, Sankt Petersburg, Izdatel'stvo RKhGA, 2013, pp. 202-215.

16. Cf. Jean-Luc Nancy: Der Eindringling. Das fremde Herz, Berlin, Merve, 2000.

17. N.T.: Todos os trechos aqui citados da obra de Nancy foram traduzidas por Pricila C. Laignier, com a colaboração de Ricardo Parente e Susan Gugenheim.

18. O título sugere um jogo de palavras com „kneading“ (amassando) and „needing“ (necessitando). 


\section{Referências}

BARTHES, Roland. (1975). The Pleasure of the Text. New York: Hill and Wang.

BLUMENBERG, Hans. (1998). Die Sorge geht über den Fluss. Frankfurt/M.: Suhrkamp.

BRANDSTETTER, Gabriele, ALBRIGHT, Ann Cooper (Org.). (2015). Choreographic Practices. Special Issue: Dis/abilities: The Politics of a Prefix, intellect journals, Volume 6, Número 1.

BRANDSTETTER, Gabriele, EGERT, Gerko, HARTUNG, Holger (Org.) (2016). Movements of Interweaving. London: Routledge.

BRANDSTETTER, Gabriele. (2013). Produktive Unsicherheiten. Kontingenz und Improvisation im Tanz. In: SAVCHUK, Valeri Savchuk, WULF, Christoph (Org.). Neopredelennost kak vyzov : media, antropologii $\square$, èstetika, Sankt Petersburg, Izdatellstvo RkhGA, p. 202-215.

COHEN, Bonnie Baindrige. (2012). Sensing, Feeling and Action. Toronto: Contact Editions.

FONTANE, Theodor. (1962). Vor dem Sturm. In: KEITEL, Walter, NÜMBERGER, Helmuth (org.). Sämtliche Werke: Romane, Erzählungen, Gedichte. Darmstadt: Wissenschaftliche Buchgesellschaft, v. 3.

KAHLERT, Christina. (2015). Klein Technique ${ }^{\mathrm{TM}}$ - Re-Patterning and Selbstkompetenz. Zur Integration somatischer Praktiken in die zeitgenössische Tanz(pädagogik)ausbildung Diss., Berlin.

KATAN, Einav. (2016). Body of Knowledge. Embodied Philosophy in Gaga, Ohad Naharin's Movement Research. London: Palgrave

NANCY, Jean-Luc. (2000). Der Eindringling. Das fremde Herz. Berlin: Merve. 
. (2008). O intruso. Tradução: Pricila C. Laignier, com a colaboração de Ricardo Parente e Susan Gugenheim.

NOWOTNY, Helga. (2016): The Cunning of Uncertainity. UK/USA: Cambridge, Polity Press.

ODENTHAL, Johannes. (2004). Entrevista. In: The Third Body - das Haus der Kulturen der Welt und die Performing Arts. ODENTHAL, Johannes, Haus der Kulturen der Welt (Org). Berlin, Theater der Zeit, p. 45-52.

SABISCH, Petra. (2002). "Körper, kontaminiert. Ein Versuch mit Randnoten zur Performance Product of Circumstances by Xavier le Roy". In: BRANDSTETTER, Gabriele, PETERS, Sibylle (Org.). De figura. Rhetorik - Bewegung - Gestalt, Munich: Fink, p. 311-326.

Recebido em: 06/09/2015

Aceito em: 10/10/2015 


\section{Artigo original}

\section{IN AND OUT OF SYNC RHYTHMS OF TRANSLATION IN PERFORMANCE AND DANCE}

Allow me to begin with a subject that has acquired a new topicality in recent weeks, namely that of "borders". The word "translation" means to "carry over", to "cross borders".

\section{Abb. 1a \& 1b}

What happens when borders are opened, overrun - or closed and sealed off again? Take Germany - and Europe - in autumn 2015. Thousands of people uprooted by wars, civil strife, and general mayhem are pouring over the borders, an estimated one million in this year alone. A human flood and mass migration which not only disregards territorial markings (Austria ends here, Germany starts here), but which also place in question all social, spatial and physical "limits". Welcoming gestures alternate with violent defensive actions. Countries' capacity to provide at short notice food, shelter and "integration" is, as the political commentators never tire of telling us, exhausted.

Everyday life, it would appear, is out of joint? Or "out of sync?" The public are faced with the challenge of absorbing this great migratory movement. There is a need for synchronization at many levels - whether temporal, physical, interpersonal or micro.

There is an air of great uncertainty which for many people acquires an existential dimension. This is more imaginary than "real". Yet this is precisely where the limits undergo a shift. And this in turn raises major questions for politics und various branches 
of science. In her new book The Cunning of Uncertainty ${ }^{1}$, which examines the search for security and the productive resources of "uncertainty", Helga Nowotny raises a key question of current sociology and scientific theory. ${ }^{2}$

The questions I am going to raise here, however, do not concern the social aspects of the synchronization of movements. After all, the dance is the ideal medium for exploring the synchronizations/ desynchronizations of movements in time and space and how they provide material for choreographies.

Before turning to the general theoretical questions concerning the interactions and rhythms of synchronized movements I should like to start with a minor case of "out of sync". The scene comes from a novel by Theodor Fontane called "Vor dem Sturm" ("Before the Storm") $)^{3}$. It is a story in which memories of Napoleon's Russian campaign are "out of sync" with the nervous state of the novel's protagonist:

Confused and distraught, the novel's protagonist, Lewin, is trying to get clear of Berlin. He arrives at a village inn called "Zur Neuen Welt" (The New World), where dance music is being played and couples are taking to the floor. He observes their movements from outside. "He kept the beat with his right foot and found the dance greatly to his taste. 'If only he could have taken part!'”, thinks Lewin. However, his inner unrest drives him on. He can still hear the strains of a "Scottish Dance" as he resumes his march, but he cannot make his tempo fit the tune. The dance rhythm interfered with the measured rigidity of his march - he was "out of sync". "As he continued on his way, Lewin, tried to match his march to the beat. For a while he succeeded, but the dance rhythm was stronger than anything else and, continuously falling out of his forced march rhythm, he proceeded in a wondrous combination of dance step and soldierly stride down the straight, poplar-lined avenue." 4

The march step breaks with the regular step. The skip step of the "Scottish Dance" (the "Écossaise") interrupts the beat of the march. And this de-synchronized movement leads hin to the 
barrier ("Schnatermanntag" (ibid.)), where he recalls the war and the defeat of Napoleon's Grande Armée. But even these images of the "shattering encounter" succumb to the dance music. The lightfooted dance rhythms of the village revels drown out the marching tread of the army and the choreography of the (lost) war. How can we understand in terms of de-synchronization the routes and encounters brought to life today by migrants worldwide? How are we to describe the transformations of spaces, collectives and social relations in terms of choreo-graphy? In a critical interpretation of Tim Ingold's concept of "Lines" Evelyn Schuler has proposed replacing and rethinking the idea of the (straight) line (which is always static and leads to exclusions) with Hans Blumenberg's ${ }^{5}$ concept of a detour.

\section{Moving, Travelling, Migrating}

To consider the relation of dance, travelling and migration means to look at forms of bodily movements on different levels and thus to ask, why and how movements influence, initiate, prevent, or change the dynamics of other movements. The field is complex. Travel and migration are undertaken for very different reasons, motivations and durations. While tourism and "recreational" travelling have increased tremendously in recent decades, resulting in one of the largest economic (and tremendously ecological) factors world-wide, there are also forms of involuntary migration, forced displacements and border crossings that take place every day due to political crises, and/ or economic developments. Migrant workers in their more or less temporary diasporas send huge amounts of money to their families worldwide on a daily basis. While travelling seems to be grounded in a sense of leaving and returning, for migratory movements the notion of "return" often remains open-ended. This has resulted in contrary evaluations of these two forms of global movements in public discourses. While travelling is usually 
highly valued, talked about, longed for, and even demanded by businesses and companies, migration is rarely seen in terms of a rich, culturally diverse background, but rather as a deficiency, a destabilizing force and a challenge to the social apparatus.

Dances and dancers do both. They travel and migrate in and between "cultures". Their global movements are shaped, influenced, enabled and sometimes prevented by the same logics/ logistics, by changing political agendas, through (institutional) invitations, visa policies, etc. Contemporary dance scene(s) are deeply connected with the economic structures that facilitate their tours and de-tours, enabling artists to collaborate globally and trans-culturally, to initiate meetings and encounters. At the same time, in contemporary dance productions these conditions find their resonances. Dance and performance have become a privileged location to critically reflect on these conditions that cause people to be "on the move". How do the ongoing global changes affect our bodies while we are coping with these new conditions?

Travelling and migrating bodies are often bodies in crisis, bodies in unstable conditions, in unfamiliar infrastructures, social constellations, often controlled by authorities while moving, and thus regulated and limited in their paths and decisions. Travelling bodies are therefore often bodies with a heightened awareness, developing tactics to adapt, to resist, to abandon or hold on to habits, ideas, and personal belongings. What kind of traces do these transits and transitions leave in the bodies, how do they re-shape its habitual ways of moving about? What kind of stories do they instigate? What kind of power structures do they reveal? How are personal and communal histories being shaped by such travels and migrations? How can we find and describe (body-) languages that adequately reflect these complex relations, transformations, these "subcutaneous" influences that (global) movements have on our various vocabularies, dispositions, gestures and daily movements?

What are the movements that emerge in the choreo-political scenes of dance and performance cultures? And how do they, in turn, interweave with movements in urban environments, and in media 
networks? The Italian-Swedish choreographer Cristina Caprioli developed in 2012 a project related to "weaving politics" which she then continued in "Choreo_Drift" (2014), a format staged in several cities in Europe, the US and the Middle East over a longer period of time, in a particularized interweaving process of dance, performance, and theory events overlapping and interrupting each other-and taking up one another's threads at different places.

The human body is its own particular arena of movements and sediments of "interweavings". This body is (in itself) not one. One might, with Roland Barthes, ask: "What body? We have several of them; the body of anatomists and physiologists, the one science sees or discusses: this is the text of grammarians, critics, commentators, philologists (the pheno-text)." ${ }^{6}$ To this, Barthes juxtaposes the "body of bliss"7: a body dissolving, dissipating and disseminating itselfthe scene of interweavings and their liquefactions, irreducible to its physiology. Barthes describes a situation which highlights these movements of weaving, resonating, and the overlapping of diverse inter-cultural and media bodies (of sound). Sitting at a bar in Tangier, Morocco, he experiences himself as a site of transit, as a multiple corporeal public space: "Music, conversations, the sounds of chairs, glasses, a whole stereophony of which a square in Tangier (as described by Severo Sarduy) is the exemplary site. That too spoke within me, and this so-called interior' speech was very like the noise of the square, like that amassing of minor voices coming to me from the outside: I myself was a public square, a sook (...)." Is the body of dancers today such a souk? A public place, where different cultures and practices of movement, body techniques and somatic experiences overlap-a polyrhythmic, stereophonic, multivoiced, multilingual concurrency and non-simultaneity of layers, whose woven patterns keep generating differences and frictions? Against the background of the expectations and experiences today's dancers are faced with in auditions, the body that comes to the fore is a scene of "interweavings" of various dance techniques and somatic practices. ${ }^{9}$ Ballet, modern dance (whether Graham, Limon, or Cunningham technique) and, along with this, release 
techniques, plus training in tai chi, yoga, experience in body-mind centering $(\mathrm{BMC})^{10}$, Alexander technique, Feldenkrais-this kind of "mix", with different emphases, often characterizes the training and processes of habitualization dancers are subjected to: intracorporeal synchronizations of body practices from very different motion and dance cultures and their often very old and standardized traditions. This poses a number of questions. How do movement techniques and their exercises of incorporation transfer themselves, interweave, or collide with one another? How, for example, do ballet training and tai chi combine with, or contradict, each other? And in what ways can these micro-corporeal synchronizations and their specific potentials become productive in terms of aesthetic design and perception?

Is what enriches the "body realities" of current and critical dance performances less the homogenization of such embodiments of multiple movement techniques than their (individual, and local, as the case may be) differentiation with all its inconsistencies? Not the icing (the shiny side) of virtuoso "techniques" but the fractures and interferences of what cannot easily be synthesized into a "body" - the very situation, that is, which performatively generates this "certain" multiple body as a permeable site and transit area of diverse and non-simultaneous "weavings" without fixating it, as described by R. Barthes: this is the focus of research and analysis on "movements of interweaving". 11

A few examples will show that, when it comes to the layering and the tensions of intra-corporeal entanglements, this can by no means be reduced to clear "types" or "formats". Rather, the fact that both differentiation and de-differentiation are becoming relevant in individual scenes and artistic works is the effect of that globalized presence of a body workshop and event culture: disseminating yoga, chi gong, flamenco, salsa, hip-hop and break-dance courses and far more techniques and practices of movement throughout the world. It is a process which has its own history. Reflection on "other" body practices than those of the poised virtuoso display goes back to the Judson Dance generation; and even further to Modernism 
in dance-which, albeit under Western colonial signature and the associated instances of self-exoticization of the (female) body, is still a scenario of weavings of entirely different social and aesthetic movement cultures and their discourses.

Artistic dance performances, in particular since the 1980s and 1990s, in their practice and their concepts, often appear to be the "product of circumstances" as regards their physically interdependent practices and different movement cultures.

A signature piece of the 1990s, Xavier Le Roy's performance of "Product of Circumstances" (1998), became the key project of a performance of the "contaminated body" (Petra Sabisch). ${ }^{12}$ The concept of contamination is almost always suggestive of the "positing" (Setzung), the connotation of impurity, if not poisoning; a practice of "synchronization" as both an intended and latent practice in dance, on the other hand, is broader in scope-also in the sense that the levels of a discourse practice and analytical reflection are implied in the concept of de/synchronizing. Examples of the wide variety and divergence of such phenomena of an embodied, intracorporeal entanglement of different body practices and traditions (singled out here from the multiplicity of the performance scene) are: the dance pieces of dancer Koffi Kôkô, whose work interweaves the origins of the shamanic body practices of his native land (Benin/ Africa), and the experiences of his training in modern dance in Paris, both in the movement patterns and in the themes of his pieces, ${ }^{13}$

\section{Abb. 2}

or Pitchet Klunchun, a dancer from Thailand, trained in classical Khon dance, who created a duo together with the Frenchman Jérôme Bel ("Pitchet Klunchun and Myself") in the format of a lecture performance; and who, in the piece "I am a Demon" (2007), ultimately connected the tradition of education in Khon dance with his adaptation of the (Western) concept of autobiographical performance, while simultaneously subverting and transforming 
his play with the "I" (already contained in the title). In what manner do these performances occupy a grey area made up of transfers of somatic practices and that which is only handed down in an authorized form, i. e. even protected as a "label": such as the trademarked Klein Technique ${ }^{\mathrm{TM}}$ training courses, ${ }^{14}$ or the "Gaga" system workshops ${ }^{15}$ developed by Ohad Naharin?

What are the new boundaries, what are the inclusions and exclusions that become effective here?

It should be noted in this context that such transfers and layerings of different cultural body practices also apply to "layers" of various historical "habits" and concepts of movement. Through them, the dancer's body becomes the site of a corporeal palimpsest-as, for instance, when Japanese dancer and Butoh pioneer Kazuo Ohno in his performances combines, bastardizes and completely reshapes various Western and Eastern dance traditions.

\section{Abb. 3}

In "Admiring la Argentina" he embodies, at the age of 78, the dance style of the stylized "Spanish dance" performed by the famous 1920s dancer "La Argentina" on her tours all around the world, including Japan. At the same time he mixes this habit of "Flamenco" with modes of movement flows from German expressive dance, which was introduced to Japan by such dancers as M. Ito and Ogai Mori, who had studied and performed in Europe. As a physical education teacher at a Japanese school, Ohno had moreover mastered the appropriate physical training and, in his encounter with Tatsumi Hijikata, he became acquainted with the latter's radical notion of a rebellion of the body as "Ankoku Butoh". Ohno transformed this concept of expressiveness by his idiosyncratic approach to Butoh, by delays and lyrical transparency-a dance of transformations through interweavings of different movement cultures and concepts of expressiveness. Also the crossing of barriers discriminating 
between the sexes, different ages of the body and between animated / inanimate became a structural characteristic of his embodiment methods. Kazuo Ohno exemplifies the fact that artistic forms of intra- and inter-corporeal de- and re-synchronizing of movement cultures and body techniques, far from leading to homogenization, are capable of contributing to a highly individual differentiation, if not to singular articulations of difference. ${ }^{16}$

Today, these developments also lead to further explorations in racism, nomadic identities, the touristic souvenir, or questions of disabilities. ${ }^{17}$ At the same time it traverses the terrain of sexual difference on the basis of corporeal transformations. What are the changes (alterations, transformations) that occur when a dancer goes through a female/male transgendering process? How does this affect the quality of their movements? These questions will be explored, as a kind of embodied "queer ethnography," by the dancer An/(J) an Kaler in a planned series of performances entitled "O.T." (the initial letters of the German words for "untitled" - tr.).

\section{Borders, Threshold Spaces, Transits}

A tour of threshold spaces, of the border zones between life and death, is offered by the performance installation "Orfeo"(after Claudio Monteverdi). It is a "rite of passage" in which the spectator passes through eight rooms linked by narrow waiting rooms - a translation - which drifts in a fixed time frame (of 10 minutes each, expanding into a time continuum) into the experience of timelessness, indeed of "the beyond".

\section{Abb. 4}

"Practice in Dying" is what the director, Susanne Kennedy, calls this installation. ${ }^{18}$ Orpheus, in search of Eurydice - that is us, the visitors to this concourse. Like Orpheus, we move in a limbo 
on the way to a world that lies "beyond": In this intermediate realm Eurydice appears. Fragments of Monteverdi's music link the phases of the tour - and her detour. The Eurydice-like performer moves through the rooms as though remote-controlled, a masked, zombielike figure trapped in a no-man's land between life and death, since Orpheus cannot let her go. The repetitive design of the rooms, the silence and the distant fragments of melody, the stereotyped femininity of the Eurydice-like figures, whose identitical masks and wigs and impassive and emotionless demeanour, whether sitting, standing, or walking, give them a "synthetic" look - all this makes it clear to the spectator that identity is in the process of "fading away".

\section{Abb. 5}

The familiar acquires an alien quality. In the expanse of time, in the midst of ennui and emptiness, all are left to their own devices, uneasily exposed to the memories, associations, thoughts of flight. Only the eyes of the masked, duplicated Eurydices move enigmatically, weirdly, concealed behind their plastic covering. And certain gestures, a way of breathing, or fidgeting in their seats as in a trance indicate that life will not be here much longer. This situation, these figures - and we ourselves - are "out of sync".

Banality and the hyperrealism of immediate proximity grant the spectator a simultaneous experience of this passage through a labyrinth of time and space. "Silence is required during the transition," 19 the Programme tells us in no uncertain times. We are admitted to a room in which "letting go" is both content and experience:

[...] Do not cling, in fondness and weakness, to this life.

Even though you cling out of weakness,

You have not the power to remain here. (see Programme). 
Instructions concerning the passage and inspired by the "Tibetan Book of the Dead", accompany the passage until the spectator in the second last, brightly gleaming room, stands directly in front of Orpheus. He is singing, intensely and in my immediate vicinity, his aria beseeching permission to stay - but only for a moment, which feels very short. Then one finds oneself standing among others at the "death bed" in which Eurydice is lying, while fragmentary recollections flicker across the screen. "The duration of this state is uncertain" (quotation from Programme).

The structure of this installation is complex and the walk through the border trooms manages to generate a high emotive potential und transformative quality. The border zones lie in a fictitious scenario. The setting invites the spectator to transfer the myth of Orpheus and Eurydice to their own experiences and expectations regarding death, dying (or "letting go"/farewell). Supported alternately by silence and music that consists of flat extended sounds (the composers have used a computer algorithm to extend Monteverdi's score to 199.5 hours), the spectator enters a state in which his experience of the liminal zone is transferred to his insides: however imagined, he feels himself in an anticipated transitional state between life and death. And the resonance - or rejection - of that duration, like some endless loop, in which the "Eurydice" figures' - already in a state of the "undead" - sit and wait, is accompanied by a feeling of change and uncertainty. Is it a productive uncertainty? ${ }^{20}$ It is a state of discomfort, a shifting of boundaries that traverse the body. The performers hardly move at all, remaining, for example, seated on the sofa. They twitch almost imperceptibly as a result of micromovements emanating from torso, diaphragm and spine as though the (vital) tone was gradually receding and the strength draining from the body. This fleeting presence, which is actually more perceptible as an absence, stirs up the spectator's emotions at the proprioceptive, kinaesthetic level. The boundaries shift between inside and outside, between ego and others (in space) - between (after-)life and nearness of death. A transition! 
In his essay "The Intruder " ("L'Intrus") ${ }^{21}$ the philosopher Jean-Luc Nancy reflects on the large theme of the "intruder" as an experience of the intrusion of death into his life, into his body.

The multishaped stranger who penetrates into my life (into my rundown, exhausted life that sometimes takes a turn for the worse and leads me to the brink of a slightly astonished resignation), is none other than death, or rather life/death(ibid., p. 27).

Nancy is writing about a heart transplant he has just undergone. It is a matter of life and death, of getting the "intruder" into the body, the inability to distinguish between what is "one's own" and what is "alien": the transplantation, the immunity and the defensive reactions of the body (p. 31). In relation to his own body, with its constantly changing borders and limits, Nancy discusses the subject of "intrusion" - a topic that has presented a constant challenge to our social and physical lives. Who is an "intruder", in what territory and in what period? And how are we to transform these experiences - the falling into an "out of sync" and the resynchronization of the collective movements of our notions of (physical, social, and political) identity?

Nancy asks if there is a general law of intrusion and offers the following answer: "There is no such thing as an absolute intrusion. As soon as a state of intrusion exists, it immediately subdivides itself, breaking down into ever new immanent distinctions" (ibid., p. 35). What the body, like an internal collective, experiences in the way of internal de-synchronizations - cardiac irregularities, extra systoles - becomes a model for the experience of intrusion and alienation in a social context. All current issues concerning borders, their violation, and the abandonment of familiar life synchronizations caused by migration, worldwide refugee movements, and "reception" culture ("hospitalité"), are affected by Nancy's philosophy of the "intruder": "The fact that one receives 
the stranger necessarily entails that one learns of his intrusion. Normally one will not want to admit this and refuse to admit him. The motive for the intrusion will itself bring about an intrusion into what we hold to be morally right and into the corrective function of morality. Even our refusal to take cognizance of and acccept an intrusion is a remarkable example of intrusion into the rightness of political correctness. Yet we cannot separate the intrusion from the truth of the stranger.

The morally right and correct assumption is that when one receives strangers their strangeness is abolished on the doorstep. It can happen, however, that one does not want to receive the stranger, and that the stranger insists and forces his way into the house anyway" (ibid., p. 9).

This paradox between the intrusion of the stranger and the abolition of his strangeness on the doorstep, is a challenge and the basis of a (trans-lation) that obviously can never "succeed". The movements that cross und describe the borders, zones and passages mentioned are therefore such as require synchronizations, de-synchronizations and re-synchronizations. Breaches, ruptures, gaps - and detours (!) - are part of these processes. In dance and performance it is those concerned with research and the fine tuning of such de-synchronizations, who have been producing important new works lately. Both in the synchronizations of collective movements, and in experiments geared to intra-body processes of polyrhythmics and temporal and physical attunings. Thus Thomas Hauert and his Compagnie ZOO, for example, in his piece "Verosimile" (2002) or in "Accords" (2008), were working with concepts based on swarm movements. The observations of the synchronizations between a collective of bodies, as "swarm", is transferred to dance formations. This is done by training in the use of somatic practices and enhanced awareness of the kinaesthetic relations between bodies and space. The constant modulation of the cohesion of the group becomes a movens of choreography. The latter is not pre-fixed, but arises at the moment of "joint movement". Their figures and time forms emerge in the course of 
the synchronization of the (group) swarm movements. One example, in which the concepts and modulations of de-synchronizations from the inner body are activated and "transferred" to the choreography, is the Trio "Kneeding", ${ }^{22}$ (2010) by Jefta van Dinther. In the generation of the dance piece he uses various somatic practices, especially body-mind centering, while the dancers use complex organ and liquid synchronizations to model ("knead") their bodies as if they were made of some kind of malleable material. They travel in their imaginations deep inside themselves, beyond the boundaries of the visible and palpable ("what you cannot touch") and arrive via this generation process at decisions on the course of choreography: nearness und distance; tempo and quality/tension of movements. The spectator is confronted with polychronically, visually und kinaesthetically distinguished, rhythmized relations between bodies, space und sound. Both de-synchronization experiments - the external one with collectives and the internal one, involving entering bodies - refer to current problems in our globalized world. "The world is out of joint," a German politician (Frank Walter Steinmeyer) remarked recently in a speech. "Out of sync". Did he mean the investigations into de-synchronizations conducted as time and motion studies in polychronic procedures and transmissions? How will this make borders crossed, extended, shifted - and re-established? If the conflict zones (whether in the social or physical context) can be internalized, if the need to drive back or expel the intruder) violently invades the comfort zones of everyday life, what then?

A straight line (in Tim Ingold's sense) does not let itself be drawn into such processes. "Out of sync" always means an abandonment of an (apparently) preordained time schedule and way of doing things. "Out of sync", however, contains the chance of a re-orientation; of a detour (Evelyn Schuler with Blumenberg) to temporary re-synchronizations of the social and individual "body" (and its networks). 


\section{Notes}

1. Helga Nowotny: The Cunning of Uncertainity, Cambridge, Polity Press, UK/ USA, 2016.

2. On the current topic of "uncertainty" cf. also the "Unsicherheit" volume published by the Zeitschrift für historische Anthropologie , Paragrana, ed. by Christoph Wulf and Jörg Zirfas, vol. 24, issue, 2015. On uncertainty and forecasts see also Gabriele Brandstetter's essay "Tanz mit dem Unvorhersehbaren: Prognosen über Bewegungen" in Gabriele Brandstetter, Sibylle Peters, Kai van Eikels (eds.) Prognosen über Bewegungen, Berlin, b_books, 2009, pp. 262-269.

3. Cf. Theodor Fontane: "Vor dem Sturm", in: Walter Keitel, Helmuth Nürnberger (eds.), Sämtliche Werke: Romane, Erzählungen, Gedichte, vol. 3, Darmstadt, Wissenschaftliche Buchgesellschaft, 1962.

4. The quotation goes on: "He passed the place where, on Schnatermanntag, he first felt the misery of the retreat. Meanwhile, he thought no more of the shattering encounter and only kept time with the music, although he had long ceased to hear it. 'Just dancing away,' he said, 'that is living. Just don't let anything get you down. The best has passed me by.'” (ibid., p. 489.)

5. Cf. in this context Blumenberg's reflections on the subject of borders, in connection with Wittgenstein's metaphor of the wall that surrounds the swamp, as described in his "Philosophische Betrachtungen" (1930). Blumenberg explains this mental image in his collection of short texts "Care Crosses the River" under the heading of "Swamp": What is crucial for him (and for our observations on the concept of "border"), is that borders are never drawn with mathematical precision. Instead they are blurry, indicating an intermediate space where inclusion and exclusion are not exactly definable. "The wall cannot be identical with the edge of the swamp. It stands round it, encloses it, while standing, as walls must do, upon a secure foundation" (p. 109). This means that the exact delineation of inexactitude is as impossible here as it is everywhere else" (p. 110). And zones/ border zones do not "have" a crossing point. They are a crossing point (ibid.); cf. Hans Blumenberg: Die Sorge geht über den Fluss, Frankfurt/M., 1988, p. 109 f.

6. Roland Barthes: The Pleasure of the Text, New York, Hill and Wang, 1975, p. 16. 
7. Ibid.

8. Roland Barthes: The Pleasure of the Text, New York, Hill and Wang, 1975, p. 49.

9. For a discussion of philosophy / aesthetics and somatic practices see Richard Shusterman: Somaesthetics - A Disciplinary Proposal, in The Journal of Aesthetics and Art Criticism, Vol. 57, No. 3. (Summer, 1999), ed. by the American Society for Aesthetics, Philadelphia, pp. 299-313.

10. Cf. Bonnie Bainbridge Cohen: Sensing, Feeling and Action, 3rd ed.,Toronto, Contact Editions, 2012.

11. Cf. Gabriele Brandstetter, Gerko Egert, Holger Hartung (eds.): Movements of Interweaving, London, Routledge, 2016, upcoming.

12. Petra Sabisch, "Körper, kontaminiert. Ein Versuch mit Randnoten zur Performance Product of Circumstances by Xavier le Roy," in Gabriele Brandstetter, Sibylle Peters (eds.), De figura. Rhetorik - Bewegung - Gestalt, Munich, Fink, 2002, pp. 311-326.

13. Interview with Johannes Odenthal, in The Third Body - das Haus der Kulturen der Welt und die Performing Arts, ed. by Johannes Odenthal and Haus der Kulturen der Welt, Berlin, Theater der Zeit, 2004, pp. 45-52.

14. Christina Kahlert: Klein Technique ${ }^{\mathrm{Tm}}$ - Re-Patterning and Selbstkompetenz. Zur Integration somatischer Praktiken in die zeitgenössische Tanz(pädagogik) ausbildung, Diss., Berlin, 2015.

15. Reference: Einav Katan: Body of Knowledge. Embodied Philosophy in Gaga, Ohad Naharin's Movement Research, London, Palgrave, 2016, upcoming.

16. On the interweaving of Butoh traditions with Western dance techniques cf. Nanako Nakajima's essay "Two Hours and One Hundred and Three Years of Dancing : Xavier Le Roy's Product of Other Circumstances and Kazuo Ohno's Butoh", in Fjord Review, Summer 2011, Volume 1, Number 3, pp. 14-19. 
17. Cf. Gabriele Brandstetter, Ann Cooper Albright (eds.): Choreographic Practices. Special Issue: Dis/abilities: The Politics of a Prefix, intellect journals, 2015, Volume 6, Number 1.

18. Martin-Gropius-Bau, Berlin, 18.09.-04.10.2015 (UA: 20.08.2015, Essen), only eight persons at a time are admitted to the complexly constructed rooms of the Museum.

19. Quotation from programme: Orfeo. Nach Claudio Monteverde - eine Sterbeübung. A production of Ruhrtriennale and the ensemble Kaleidoskop, coproduced by Berliner Festspiele und Toneelgroep Oostpool, 2015.

20. Cf. above: G. Brandstetter: „Produktive Unsicherheiten. Kontingenz und Improvisation im Tanz", in: Valeriǐ Savchuk, Christoph Wulf (eds.), Neopredelennost' kak vyzov : media, antropologïa, èstetika, Sankt Petersburg, Izdatel'stvo RKhGA, 2013, pp. 202-215.

21. Cf. Jean-Luc Nancy: Der Eindringling. Das fremde Herz, Berlin, Merve, 2000.

22. The title implies a word-play with „kneading“ and „needing“. 